\title{
Embolisation of recently ruptured intracranial aneurysms
}

\author{
J V Byrne, A J Molyneux, R P Brennan, S A Renowden
}

\begin{abstract}
Patients with subarachnoid haemorrhage due to the rupture of aneurysms unsuitable for craniotomy and clipping have been treated by coil embolisation within three weeks. Sixty nine of 75 consecutive patients were sucessfully treated. Procedure related complications occurred in 10 patients, resulting in permanent neurological deficits in three and one death $(4 \cdot 8 \%)$. The Glasgow outcome scores at six weeks were 53 grade 1 , seven grade 2, four grade 3, and five grade 5 . These results are comparable with surgical series despite a high proportion of aneurysms in the posterior cerebral circulation.
\end{abstract}

$(\Im$ Neurol Neurosurg Psychiatry 1995;59:616-620)

Keywords: Intracranial aneurysm; embolisation; coils

The annual incidence of aneurysmal subarachnoid haemorrrhage in the United Kingdom is 10-15 per $100000^{1}$ with a mortality of $30-40 \%$. Rebleeding carries a similarly high mortality and occurs in up to $29 \%$ of patients in the first three weeks, ${ }^{2}$ falling to an annual rate of about $3 \%$ six months after subarachnoid haemorrrhage. ${ }^{3}$ To prevent rebleeding, early craniotomy and surgical clipping is advocated to isolate the ruptured aneurysms from the intracranial circulation.

The timing of craniotomy remains controversial but is usually performed either within two to three days of ictus or delayed until at least the 10th day to avoid the period when patients are most vulnerable from the effects of cerebral vasospasm after subarachnoid haemorrhage. ${ }^{4}$ Endovascular treatment-that is, catheter based techniques for embolisationare particularly attractive in this acute period but their safety has yet to be established. Early endovascular attempts to induce aneurysm thrombosis involved parent artery occlusion ${ }^{5}$ or packing the lumen with latex or silicon balloons. ${ }^{67}$ More recently, thrombogenic metal coils have been used as they are simpler to deploy and safer because they deform more easily to the shape of the lumen of the aneurysm. ${ }^{8}$ We report a group of patients treated by endosaccular packing with platinum coils soon after aneurysm rupture.

Materials and methods

Between September 1992 and October 1994, 75 consecutive patients (mean age $53 \cdot 2$, range
31-72 years) were referred for endovascular treatment within three weeks of documented subarachnoid haemorrrhage. These patients were referred because surgical clipping had either proved impossible at craniotomy (three patients) or was considered unsuitable due to the location (36 patients), size (16 patients), or wall thickness of the aneurysm (three patients) or because the patient was unfit for craniotomy (17 patients). This assessment was made by a consultant neurosurgeon, usually the referrer, in all the patients. Coincidental second aneurysms were treated in eight of the patients: to avoid the need for bilateral craniotomy in two patients with multiple aneurysms, because of anticipated surgical difficulty in two patients, and at the request of four patients. Endovascular treatment was attempted in 83 aneurysms. The commonest site was the terminal basilar artery (36/83); table 1 gives the aneurysm locations. There were 43 small, 38 large, and two giant aneurysms defined by a maximum dimension $<10 \mathrm{~mm}$, $<25 \mathrm{~mm}$, or $\geqslant 25 \mathrm{~mm}$.

The procedures were normally conducted under general anaesthesia. Initially some patients were treated under local anaesthesia if they were well enough to cooperate but latterly all patients were treated under general anaesthesia. The aneurysms were catheterised with a specially adapted variable stiffness microcatheter (Tracker, Target Therapeutics) introduced through a larger catheter placed in the appropriate carotid or vertebral artery and packed with electrolytically detached platinum coils (Guglielmi detachable coils, Target Therapeutics). All but two of the procedures were performed via a percutaneous femoral artery puncture. In two patients percutaneous

Table 1 Sites of aneurysms

\begin{tabular}{lcc}
\hline Parent artery & $\begin{array}{c}\text { Treatment } \\
\text { attempted }\end{array}$ & $\begin{array}{c}\text { Treatment } \\
\text { completed }\end{array}$ \\
\hline Anterior cerebral: & 1 & 1 \\
$\quad$ Pericallosal & 1 & 12 \\
Communicating & 14 & 14 \\
Middle cerebral: & 14 & 7 \\
Bifurcation & & 5 \\
Internal carotid: & 7 & 4 \\
$\quad$ Permination & 6 & 1 \\
PCoA & 5 & 23 \\
Ophthalmic & 1 & 6 \\
Posterior cerebral & 25 & 3 \\
Basilar: & 6 & 1 \\
$\quad$ Termination & 3 & 77 \\
Trunk & 1 & \\
Vertebral: & 83 & \\
Trunk & &
\end{tabular}

PCoA = Posteror communicating artery; PICA = posterior inferior cerebellar artery. 
common carotid artery puncture was performed because tortuous proximal arteries prevented catheterisation of the carotid artery from the groin. To reduce the risk of thromboembolism during and after coil placement patients were anticoagulated by intravenous bolus injections of heparin (prescribed to maintain the activated thromboplastin time at two to three times baseline levels). In this regard our protocol evolved during the period of the study. Initially only perioperative anticoagulation was given but now patients are given heparin by intravenous infusion for 24 hours after embolisation and prescribed oral aspirin ( $75 \mathrm{mg}$ daily) for three months.

The technique used for endosaccular packing and electrolytic detachment of platinum coils has been previously described. ${ }^{10}$ The aneurysms were packed to occlude as much of their lumen as possible while preserving patency of the parent-that is, the artery of origin and adjacent arteries. Clinical examinations of the patients performed immediately before and 48 hours after treatment were scored according to the World Federation of Neurological Surgeons grading scale. ${ }^{11}$ An assessment of outcome was made six weeks after treatment using a modified Glasgow outcome scale ${ }^{12}$ scored as follows: $1=$ good recovery, fully independent, $2=$ moderately disabled but independent, $3=$ severely disabled, $4=$ vegetative survival, and $5=$ dead. Outcome assessments were based on outpatients reviews or telephone enquiry of the patient or their carers.

\section{Results}

Six of the 75 patients referred for endovascular treatment were not treated because the neck of the aneurysm was too wide to retain coils (three patients) or the proximal vessels were so tortuous that the aneurysm could not be catheterised (two patients) and the procedure was abandoned in one patient after catheterisation of the aneurysm displaced intraluminal thrombus causing a distal embolus. The remaining 69 patients underwent 83 procedures to occlude 77 aneurysms (six patients being treated twice; four for second aneurysms). Thirty three patients were treated in the first, 22 in the second, and 14 in the third week after the last subarachnoid haemorrhage. Table 2 shows the intervals between haemorrhage and treatment. Complete occlusion was achieved in $61(79 \%)$. Incomplete occlusion of the remaining 16 aneurysms con- sisted of a neck remnant only in 13 and residual filling of up to $10 \%$ of the original lumen in three aneurysms.

This result was achieved with preservation of parent arteries except in three instances. On two occasions pressure from the packed aneurysm occluded adjacent vessels and in one patient, treated for a posterior inferior cerebellar artery aneurysm, satisfactory embolisation required occlusion of the distal vertebral artery. No symptoms resulted from these occlusions, presumably because the arterial territories involved had already developed a collateral blood supply.

Procedure related complications occurred in 10 patients $(11 \%)$, resulting in three permanent neurological deficits and one death. These occurred during or immediately after treatment. Six patients developed new neurological deficits caused by distal thromboemboli attributable to catheter or coil manipulations. Two patients recovered within days, one after two months, and three have residual deficits, including the patient in whom the procedure was abandoned.

Three aneurysms ruptured during coil placement. This situation was managed by continuing to deploy coils in two patients and immediate craniotomy and clipping in one patient. Two patients made good recoveries and one died two weeks later after rehaemorrhage (see later). One further patient had a fatal subarachnoid haemorrhage immediately after embolisation which was assumed to be due to rupture of an adjacent untreated aneurysm.

In the 48 hours after successful treatment the grades of 48 patients $(69.5 \%)$ were unchanged, 15 patients (22\%) had improved grades, and six patients deterioriated (four by one grade, one by two grades, and one patient died). Table 3 presents the immediate outcomes.

Six patients developed ischaemic symptoms attributable to spontaneous thromboemboli or vasospasm in the first week after treatment.

Table 3 WFNS grade before and 48 hours after embolisation

\begin{tabular}{llrrrr}
\hline & \multicolumn{5}{l}{ Post-treatment } \\
\cline { 3 - 6 } Grade & Pretreatment & \multicolumn{1}{c}{$I$} & \multicolumn{1}{c}{$I I$} & $I I I$ & $I V$ \\
\hline I & 20 & 17 & 1 & 1 & 0 \\
II & 27 & 7 & 18 & 2 & 0 \\
III & 18 & 1 & 6 & 10 & 1 \\
IV & 4 & 0 & 0 & 1 & 3 \\
Total & 69 & 25 & 25 & 14 & 4 \\
\hline
\end{tabular}

Table 4 Outcome scores six weeks after treatment compared with pretreatment grade

\begin{tabular}{lllrrr}
\hline \multirow{2}{*}{$\begin{array}{l}\text { Outcome } \\
\text { scores }\end{array}$} & $\begin{array}{l}\text { No of } \\
\text { treated } \\
\text { patients }\end{array}$ & \multicolumn{4}{l}{ Pretreatment grades } \\
\cline { 2 - 6 } & \multicolumn{1}{c}{$I$} & $I I$ & $I I I$ & $I V$ \\
\hline 1 & 53 & 18 & 22 & 12 & 1 \\
2 & 7 & 1 & 4 & 2 & 0 \\
3 & 4 & 0 & 0 & 2 & 2 \\
4 & 0 & 0 & 0 & 0 & 0 \\
5 & 5 & 1 & 1 & 2 & 1 \\
Total & 69 & 20 & 27 & 18 & 4 \\
\hline
\end{tabular}

and treatment

\begin{tabular}{|c|c|c|c|c|c|c|c|c|c|c|c|c|}
\hline \multirow[b]{2}{*}{ Grade } & \multicolumn{3}{|c|}{ Day 1} & \multicolumn{3}{|c|}{ Day 2-3 } & \multicolumn{3}{|c|}{ Day 4-7 } & \multicolumn{3}{|c|}{ Day 8-21 } \\
\hline & $n$ & Pred & Actual & $n$ & Pred & Actual & $n$ & Pred & Actual & $n$ & Pred & Actual \\
\hline $\begin{array}{l}1 \\
2 \\
3 \\
4\end{array}$ & $\begin{array}{l}1 \\
0 \\
1 \\
1\end{array}$ & $\begin{array}{l}0 \cdot 7 \\
0 \\
0 \cdot 5 \\
0 \cdot 4\end{array}$ & $\begin{array}{l}1 \\
0 \\
1 \\
1\end{array}$ & $\begin{array}{l}1 \\
5 \\
6 \\
0\end{array}$ & $\begin{array}{l}0.85 \\
3 \cdot 75 \\
3 \cdot 6 \\
0\end{array}$ & $\begin{array}{l}1 \\
5 \\
6 \\
0\end{array}$ & $\begin{array}{l}6 \\
5 \\
6 \\
1\end{array}$ & $\begin{array}{l}5 \cdot 7 \\
4 \\
4 \cdot 2 \\
0 \cdot 5\end{array}$ & $\begin{array}{l}6 \\
5 \\
4 \\
1\end{array}$ & $\begin{array}{r}12 \\
17 \\
5 \\
2\end{array}$ & $\begin{array}{l}12 \\
16 \cdot 15 \\
4 \\
1 \cdot 1\end{array}$ & $\begin{array}{r}11 \\
16 \\
5 \\
1\end{array}$ \\
\hline Total & 3 & 1.6 & 3 & 12 & $8 \cdot 2$ & 12 & 18 & $14 \cdot 4$ & 16 & 36 & $33 \cdot 25$ & 33 \\
\hline
\end{tabular}

$\mathbf{n}=$ Number of treated patients; pred = predicted survivors according to grade at time of treatmen and interval sinc 
(A) Subtraction angiogram showing an aneurysm of the right middle cerebral artery with vasospasm affecting the supraclinoid internal the proximal anterior, and the middle cerebral arteries. (B) Subtraction angiogram during coil embolisation. $A$ coil has been delivered into the aneurysm via a microcatheter despite the presence of vasospasm. (C) Follow up angiogram eight months after treatment and recovery showing complete occlusion of the aneurysm.

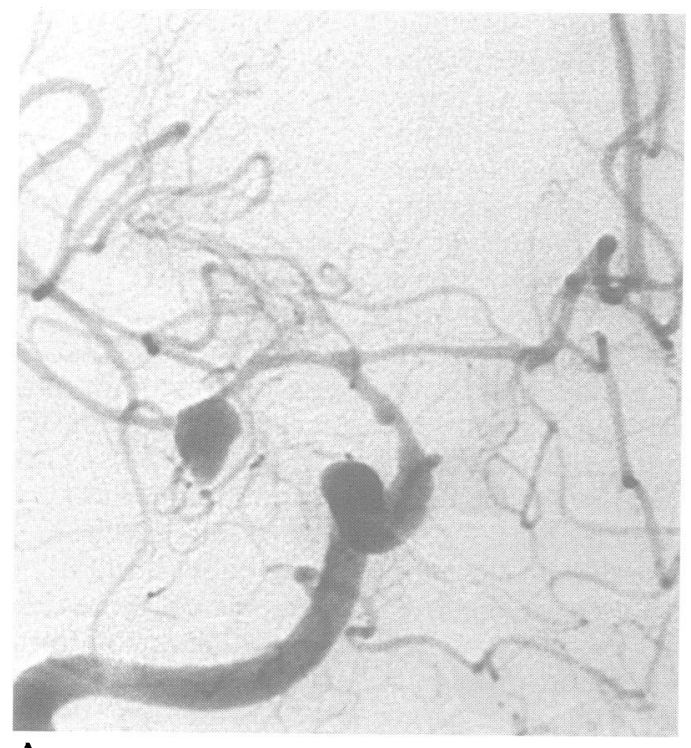

A

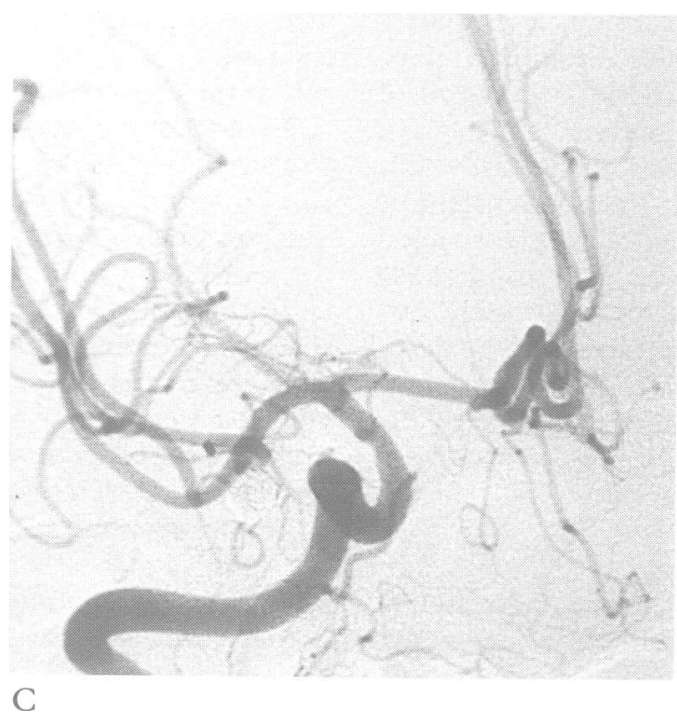

These included hemiparesis, dysphasia, hemianopsia, and fluctuating unconsciousness. Other causes for these symptoms, such as rehaemorrhage or hydrocephalus were excluded by CT. These patients were treated using antispasmodic, anticoagulant, and antiplatelet drugs in various combinations despite which deficits remained six weeks later in two patients and two patients died. Documented rebleeding occurred in two patients and both died. The first patient bled two weeks after an embolisation which had been complicated by perforation of the aneurysm and the second patient bled from a coincidental middle cerebral aneurysm while awaiting elective surgical clipping, having made a good recovery after embolisation of a basilar aneurysm.

Four additional patients experienced transient neurological symptoms one to four weeks after embolisation. These lasted minutes and resolved completely within hours. They included visual disturbance, hemiparesis, and brief periods of unconsciousness. Three of the patients had already left hospital and were not examined during these episodes but all responded to low dose aspirin treatment or long term anticoagulation with warfarin.

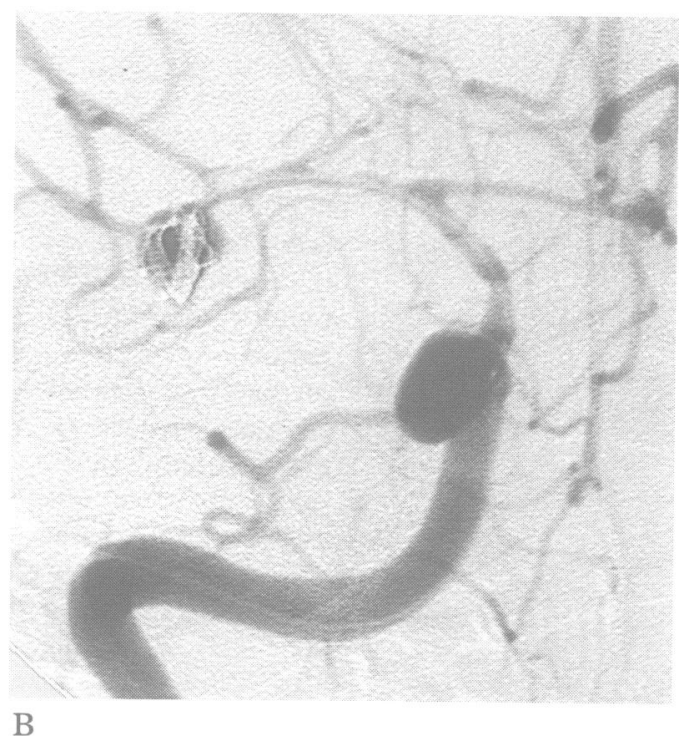

Patients with aneurysms arising in the anterior circulation experienced adverse events, both immediate and delayed, as often as patients with aneurysms of the posterior circulation (10 each).

At the time of assessment six weeks after sucessful treatment five patients $(7 \%)$ had died and five patients ( $7 \%$ ) had persistent focal neurological deficits from the causes detailed above. Table 4 gives the outcome assessments together with pretreatment clinical grades. Therefore 60 patients $(87 \%)$ made good recoveries (grades 1 and 2) and none of the patients survived in a vegetative state.

\section{Discussion}

The principles of patient management after subarachnoid haemorrhage are to provide the best possible conditions for recovery and prevention, and if necessary treatment complications such as rebleeding, hydrocephalus, or cerebral artery vasospasm. Surgical procedures to secure aneurysms against rerupture or to reduce intracranial pressure due to CSF flow obstruction have evolved in order not to jeopardise recovery. The endovascular route can in theory prevent rebleeding by isolating the aneurysm from the circulation with less trauma than craniotomy and clipping. To realise this potential, embolisation must be effective in preventing rebleeding. It should also be applicable to as many patients as clipping and as safe or safer than conventional surgical techniques.

If aneurysms are completely occluded by embolisation then the technique should be as effective as surgical clipping at protecting patients against rehaemorrhage. In the short term it seems to provide such protection as no rebleeding occurred from aneurysms completely occluded by coils in out patients or those of Casasco et al. ${ }^{9}$ However, rebleeding occurred in one of our patients and three of 11 patients with incompletely occluded aneurysms in the series of Casasco et al. So partial treatment cannot be considered protective in the short term. It remains to be seen 
if embolisation is as effective as surgical clipping in preventing aneurysm rebleeding ${ }^{1314}$ or regrowth ${ }^{15}$ in the long term.

It is difficult to estimate whether embolisation will be applicable to more or less patients then conventional surgery without a comparative study. In a large surgical series $17 \%$ of patients entering the study after subarachnoid haemorrhage were not treated surgically and of those that underwent craniotomy $6 \%$ of the aneurysms could not be clipped. ${ }^{16}$ All our patients were referred because of established or anticipated difficulties in clipping which implies that embolisation will be applicable to more patients than surgery, but $8 \%$ of patients could not be treated. The two approaches have different technical constraints. For instance, catheterisation and therefore treatment of aneurysms in the posterior circulation was often easier than aneurysms in the anterior circulation because the proximal vessels were less tortuous whereas the converse usually applies for their exposure at craniotomy. Likewise, narrowing of vessels proximal to the aneurysms due to vasospasm, which was evident on angiography at the time of treatment in 23 patients, did not on its own prevent catheterisation and the fine microcatheters used do not obviously reduce blood flow in such arteries (figure). There remains, however, a small proportion of patients who cannot be treated by embolisation because the neck of the aneurysm is too wide to retain coils, proximal arteries are so tortuous that the aneurysm cannot be catheterised, or because the local anatomy precludes safe embolisation. The endovascular therapist needs to obtain a clear view of the neck of the aneurysm and the adjacent arteries to ensure that coils are contained solely within the lumen of the aneurysm. This is not always possible.

Coil embolisation of intracranial aneurysms is a relatively new technique and our perioperative and postoperative management protocol has evolved during the period of the study. Initially, only patients who were unable to cooperate were treated under general anaesthesia, which included most patients treated acutely after subarachnoid haemorrhage. Latterly, all patients treated within three weeks of the haemorrhage have been treated under general anaesthesia because aneurysm rupture during embolisation has occurred exclusively in such patients and this complication is better managed in the anaesthetised patient. The commonest procedure-related complication, however, was stroke due to the formation of inappropriate intra-arterial thrombus. In response, we have increased the dose and duration of prophylactic heparin treatment. Currently intravenous heparin is given during intracranial manipulation and continued for a minimum of 24 hours after the aneurysm has been occluded. Intravenous heparin is used because its effect can be easily reversed should patients require a neurosurgical procedure such as CSF shunting. Prophylactic antiplatelet therapy is less easily reversed and therefore given only during the convalescent period when some patients have developed transient neurological events which we attribute to emboli originating from the thrombosed aneurysm neck.

Symptoms of cerebral ischaemia due to thromboembolic complications may 'be difficult to distinguish from other causes in the subacute phase after subarachnoid haemorrhage. The occurrence and morbidity associated with vasospasm depends on the volume and extent of the original haemorrhage. ${ }^{1718}$ It is possible that catheterisation might initiate or exacerbate arterial vasospasm but transcranial Doppler monitoring of cerebral blood velocity performed during embolisation in 20 patients did not show any effect during or after endosaccular packing with coils. ${ }^{19}$ Furthermore, most patients' clinical grade had improved at 48 hours after treatment and the outcomes at six weeks are comparable with those after surgical management of all aneurysms ${ }^{20}$ and with the best results reported for the treatment of vertebrobasilar aneurysms. ${ }^{21}$

A potentially serious consequence of avoiding craniotomy is that no visual inspection is made to confirm that the putative aneurysm had recently ruptured. The endovascular therapist has, therefore, to rely on symptoms, signs, and imaging to decide in patients with multiple aneurysms, which has ruptured. Another advantage claimed for exposure of the aneurysm at craniotomy is that it is easier to control intraoperative bleeding due to aneurysm rupture. That may well be true, but the consequences of rupture during endosaccular packing are not as dire as might be imagined, because the operator can stop the bleeding by occluding the aneurysm lumen with coils and as aneurysms are not exposed to atmospheric pressure, intracranial pressure acts to tamponade bleeding.

The long term security provided by coil embolisation in preventing rebleeding or regrowth of aneurysms remains unknown. The technique seems to improve survival rates, however, presumably by protecting patients from rebleeding during the first few weeks after aneurysm rupture. Analysing our data using the method of Alvord et al ${ }^{22}$ for predicing survival after subarachnoid haemorrhage, 57.45 patients would survive untreated to two months (table 4). Sixty four of our patients survived, as there were no additional deaths between six weeks and two months of treatment. The ratio of actual to predicted survivors as a percentage was therefore $111.5 \%$, which compared with means of $98.8 \%$ for medical and $102 \cdot 8 \%$ for surgical treatments analysed in 1972. Comparison with surgical treatment is difficult because our patients were referred because of anticipated surgical difficulties and results have improved over the last 20 years. It remains to be seen whether the endovascular route is safer than conventional surgery for operable aneurysms and in this respect the role of embolisation will be best assessed in studies comparing contemporary surgical and endovascular treatment methods. 
1 Brewis M, Poskanzer DC, Rolland C, Miller H Neurological disease in an English city. Acta Neuro Scand (suppl) 1966;42(24):1-89.

2 Locksley HB. Report on the Cooperative study of Intracranial Aneurysms and Subarachnoid Haemorrhage: section V. Part II. Natural history of subarachnoid haemorrhage, intracranial aneurysms and arteriovenous malformations. Based on 6368 cases in the cooperative study. F Neurosurg 1966;25:321-68.

3 Winn HR, Richardson AE, Jane JA. The long-term prognosis in untreated cerebral aneurysms. 1. Incidence of late haemorrhage in cerebral aneurysms: a 10 year evaluation of 364 patients. Ann Neurol 1977;1:358-70

4 Ohman J, Heiskanen O. Timing of operation for ruptured supratentorial aneurysms: a prospective randomized study. 7 Neurosurg 1989;70:55-60.

5 Serbinenko FA. Balloon Catheterisation and occlusion of major cerebral vessels. $f$ Neurosurg 1974;41 125-45.

6 Debrun G, Fox A, Drake C, et al. Giant unclippable aneurysms: treatment with detachable balloons. AfNR $1981 ; 2: 167-73$

7 Higashida RT, Halbach VV, Barnwell SL, et al. Treatment of intracranial aneurysms with preservation of the parent vessel: results of percutaneous balloon embolisation in 84 patients. AFNR 1990;11:633-40.

8 Hilal SK, Khandii A, Solomon RW. Obliteration of intracranial aneurysms with pre-shaped highly thrombogenic cranial aneurysms with pre-shaped highly thrombogenic
coils. Radiology 1989;173:250-7.

Cas. RE $A$, et al. Selective endovascular treatment of 71 intracrania aneurysms with platinum coils. F Neurosurg 1993;73: 3-10.

10 Guglielmi G, Vinuela F, Dion J, et al. Electrothrombosis of saccular aneurysms via endovascular approach Part II: preliminary clinical experience. F Neurosurg 1991;75: 8-14

11 Drake CG. Report of World Federation of Neurological
Surgeons Committee on a universal subarachnoid haemorrhage grading scale. $\mathcal{F}$ Neurosurg 1988;68:985-6.

12 Jennett B, Bond $M$. Assessment of outcome after severe brain damage. A practical scale. Lancet $1975 ; \mathrm{i}: 480-4$.

13 Feuerberg I, Lindquist C, Lindquist $M$, et al. Natural history of postoperative aneurysm rests. $\mathcal{F}$ Neurosurg 1987; 66:30-4.

14 Sakaki $T$, Takeshima $T$, Tominago $M$, et al Recurrence of ICA-PCoA aneurysms after neck clipping. $₹$ Neurosurg ICA-PCoA ane

15 Lin T, Fox AT, Drake CG. Regrowth of aneurysm sacs from residual neck following aneurysm clipping. f Neurosurg 1989;70:556-60.

16 Kassel NF, Torner TC, Horley EC, et al. The international cooperative study on the timing of aneurysm surgery. Part I: overall management results. $f$ Neurosurg 1990;73: 18-36.

17 Fisher CM, Kistler JP, Davis JM. Relation of cerebral vasospasm to subarachnoid haemorrhage visualized by computerized tomographic scanning. Neurosurgery 1980 ; 6:1-8.

18 Mohsen F, Pomonis S, Illingworth R. Prediction of delayed cerebral ischaemia after subarachnoid haemorrhage by
computed tomography. $\mathcal{f}$ Neurol Neurosurg Psychiatry 1984;47:1197-202.

19 Rowe JG, Byrne JV, Molyneux AJ, et al. Haemodynamic consequences of embolising aneurysms: a transcranial Doppler study. Br 7 Neurosurg 1995 (in press)

20 Kassel NF, Torner JC, Jane JA, et al. The International Cooperative Study on the Timing of Aneurysm Surgery. Part 2: surgical results. $\mathcal{F}$ Neurosurg 1990;73:37-47.

21 Peerless SJ, Hernesniemi JA, Gutman FB, Drake CG. Early surgery for ruptured vertebrobasilar aneurysms. $\mathcal{F}$ Neurosurg 1994;80:643-9.

22 Alvord EC, Loeser JD, Bailey WL, et al. Subarachnoid haemorrhage due to ruptured aneurysms. A simple method of estimating prognosis. Arch Neurol 1972;27: 273-84. 